\title{
Characterization and determination of the thermodynamic and kinetic properties of the adsorption of the molybdenum(VI)-calmagite complex onto active carbon
}

\author{
Sérgio L.C. Ferreira, ${ }^{*}$ Heloysa M.C. Andrade, and Hilda C. dos Santos \\ Universidade Federal da Bahia, Campus Universitário de Ondina, 40170-290 Salvador, Bahia, Brazil
}

Received 16 May 2003; accepted 29 July 2003

\begin{abstract}
The extraction of the Mo(VI)-calmagite complex by adsorption onto active carbon is investigated in the present paper. Thermodynamic and kinetic properties and different adsorption isotherms were determined. The adsorption studies have shown that both the empirical Freundlich and the classical Langmuir isotherms successfully fit the experimental results. The overall adsorption process was exothermic and spontaneous in the temperature range from 283 to $323 \mathrm{~K}$; namely, $\Delta H^{0}$ and $\Delta S^{0}$ values were found to be $-8.15 \mathrm{~kJ}$ mol ${ }^{-1}$ and $-3.86 \mathrm{~J} \mathrm{~mol}^{-1} \mathrm{~K}$, respectively. The high activation energy demanded for desorption of the Mo(VI)-calmagite complex surface indicated that the overall process was controlled by the slow desorption, while a fast and low activated adsorption of the complex onto active carbon surface was fairly favored. These features indicate that active carbon may be very useful for preconcentration of molybdenum from diluted solutions.
\end{abstract}

(c) 2003 Elsevier Inc. All rights reserved.

\section{Introduction}

Active carbons are among the most used sorbents for separation and preconcentration procedures for metal ion determination by spectroanalytical techniques such as inductively coupled plasma with optical emission spectrometry (ICP-OES) and flame atomic absorption spectrometry (FAAS). They are characterized by high adsorption capacity, allowing application under broad preconcentration conditions. Active carbons have often been proposed for molybdenum determination, including batch operation [1-4] and off-line column procedures [5]. However, the driving parameters for the adsorption phenomena and the related kinetic and thermodynamic properties are seldom characterized. Okutani and co-workers have determined the Freundlich isotherm for the extraction of indium(III) [6] and beryllium(II) [7] onto active carbon as acetylacetonate complexes. Saeed et al. [8-11] and Hasany et al. [12-15] have carried out systematic investigations on the processes of adsorption of several metal complexes onto

\footnotetext{
* Corresponding author.

E-mail address: slcf@ufba.br (S.L.C. Ferreira).
}

polyurethane foam, including the determination of the main kinetic and thermodynamic properties, namely, $\Delta H, \Delta S$, and $\Delta G$.

In the present paper, the extraction of molybdenum(VI) ions as calmagite (1-(1-hydroxy-4-methyl-2-phenylazo)2-naphtol-4-sulfonic acid) complexes onto active carbon has been investigated. Thermodynamic and kinetic properties were determined from the adsorption isotherms. A previous paper [1] has established a procedure for molybdenum determination in geological matrices and seawater by ICP-OES, after separation and preconcentration using calmagite and active carbon. The use of calmagite as reagent for molybdenum determination was proposed by Mushran et al. [16].

Active carbons are highly porous materials, attaining porosity as high as $80-85 \%$. In spite of this, the pore size distribution may be very complex for these materials and some pores are so small that the available pore volume will depend on the size of the different molecules diffusing into the porous carbon structure [17]. Consequently, mass transfer limitations may control the metal extraction by adsorption of metal complexes. On the other hand, the nature of the surface functional groups on the active carbon may also play an important role governing the adsorption process. 
Table 1

Operating parameters for the inductively coupled plasma spectrometer

\begin{tabular}{lc}
\hline Incident output power & $650 \mathrm{~W}$ \\
Reflected power & $<5 \mathrm{~W}$ \\
Nebulizer & Glass, Meinhard \\
Plasma gas flow rate & $7.51 \mathrm{~min}^{-1}$ \\
Auxiliary gas flow rate & $0.81 \mathrm{~min}^{-1}$ \\
Aerosol carrier gas flow & $0.81 \mathrm{ml} \mathrm{min}^{-1}$ \\
Solution uptake rate & $2.51 \mathrm{~min}^{-1}$ \\
Signal integration time & $5 \mathrm{~s}$ \\
Integration for determination & 3 \\
\hline
\end{tabular}

\section{Experimental}

\subsection{Apparatus}

A sequential inductively coupled atomic plasma spectrometer, Applied Research Laboratories Model 3410 minitorch, with an IBM PC computer was used. The emission intensity measurements were made under the conditions shown in Table 1 . The calibration graphs $\left(0-4.0 \mu \mathrm{g} \mathrm{ml}^{-1}\right)$ for molybdenum were built using solutions prepared from $1 \mathrm{mg} \mathrm{ml}^{-1}$ stock solution.

A 300 ANALYSER pH meter was used to measure the $\mathrm{pH}$ values.

A VKS-100 mechanical shaker was used to homogenize the suspensions at $100 \mathrm{cpm}$.

\subsection{Reagents}

All reagents were of analytical grade unless otherwise stated. Double-distilled water was used to prepare all solutions. Nitric and hydrochloric acids were of Suprapur quality (Merck). Laboratory glassware was kept overnight in a $10 \%$ nitric acid solution. Before use the glassware was rinsed with deionized water and dried in a dust-free environment.

Molybdenum solution $\left(5.00 \mu \mathrm{g} \mathrm{ml}^{-1}\right)$ was prepared by diluting a $1000 \mu \mathrm{g} \mathrm{ml}^{-1}$ molybdenum solution (Merck).

Calmagite solution $(0.15 \%)$ was prepared by dissolving $0.30 \mathrm{~g}$ (Merck) in demineralized water.

The active carbon adsorbent (100-150 mesh) was provided by Aldrich and was used for the preconcentration experiments without any pretreatment. The specific surface area of the sample was determined from nitrogen adsorption isotherms, using the BET method, and it was found to be $1513 \mathrm{~m}^{2} \mathrm{~g}^{-1}$. The DRIFT spectra of the active carbon revealed bands assigned to conjugated polyaromatic compounds and the absorption spectra in the IR region of chemisorbed pyridine indicated the presence of slightly acid surface sites.

\subsection{Procedure}

Aliquots of $50 \mathrm{ml}$ of solution containing molybdenum(VI) ions $(25.0 \mu \mathrm{g})$ were added to stoppered flasks in a thermalized water bath. The $\mathrm{pH}$ was adjusted to 2.0 by adding $1 \mathrm{moll}^{-1}$ hydrochloric acid. Then $5 \mathrm{ml}$ of $0.10 \%$ calmagite solution was added. After shaking, a mass of $50 \mathrm{mg}$ of activated carbon was added and the mixture was further shaken for $5 \mathrm{~min}$. The system was then filtered under vacuum through a $2.5-\mathrm{cm}$-diameter filter paper (Whattman No. 40) set on a glass crucible. The filtered residue of active carbon was transferred to polypropylene flask and digested with $2.00 \mathrm{ml}$ of concentrated nitric acid solution in a sand bath at $120^{\circ} \mathrm{C}$ until completely dry. The residue was treated with $5 \mathrm{ml}$ of a $5 \%(\mathrm{v} / \mathrm{v})$ nitric acid solution up to the mark and the resulting solution was taken for molybdenum determination by inductively coupled plasma optical emission spectrometry.

\section{Results and discussion}

\subsection{Adsorption studies}

Previous adsorption experiments were performed in order to properly determine the conditions for the present physicochemical investigation and are detailed elsewhere [1]. The amount of sorbed complex was expressed as the sorption percent, $\% S$,

$\% S=\frac{C_{0}-C_{e}}{C_{0}} \times 100$,

where $C_{0}$ and $C_{e}$ correspond to the complex concentration in solution respectively before and after shaking. The sorption percent is correlated to the distribution ratio by the equation [6-14]

$k_{d}=\frac{C_{0}-C_{e}}{C_{e}} \frac{V}{W}$,

where $V$ is the volume of solution $\left(\mathrm{cm}^{3}\right)$ and $W$ is the weight of active carbon in equilibrium. Thus,

$S \%=\frac{100 k_{d}}{k_{d}+V / W}$.

Accordingly, the sorption of the Mo(VI)-calmagite complex was quantitative and constant in the $\mathrm{pH}$ range 1.0-3.0. Controlling the $\mathrm{pH}$ with hydrochloric acid and acetate buffer, $96 \pm 4 \%$ sorption was attained at $\mathrm{pH} 2.0$ and $298 \mathrm{~K}$, corresponding to a distribution coefficient $k_{d}=2.4 \times 10^{4}$ $\mathrm{cm}^{3} \mathrm{~g}^{-1}$. The high $k_{d}$ value indicated that the sorption of the Mo(VI)-calmagite complex was highly favored and the molybdenum-calmagite reaction was highly selective at pH 2.0 [1].

\subsection{Adsorption isotherms}

The original derivation of the Langmuir adsorption model considered only forces between adsorbate and substrate, leading to a monolayer of an ideal gas chemisorbed onto distinct sites of a homogeneous surface. As a consequence, the adsorption enthalpy ( $\left.\Delta H_{\text {ads }}\right)$ should be constant for all 
sites, independent of the surface coverage [18]. In solute adsorption from solution, substrate-solute, substrate-solvent, solvent-solute, and solvent-solvent interactions and possibly other forces are involved and therefore a complete theoretical description is highly complex [19]. In spite of this, Giles et al. $[19,20]$ have proposed a simplified treatment, showing theoretical and experimental evidence for a consistent fitting of the solute adsorption data to the Langmuir isotherm. Thus, the kinetics of the solute adsorption from solution is considered to be similar to that in gas adsorption, nevertheless allowing for differences in the free path of the molecules [19,21].

From a kinetic derivation and considering a solutesolvent system where only one component of the liquid solution (adsorbate) significantly adsorbs onto the solid surface (substrate or adsorbent), the Langmuir adsorption isotherm may be expressed as

$\frac{C_{e}}{C_{\mathrm{ads}}}=\frac{1}{K_{L} b}+\frac{C_{e}}{K_{L}}$,

where $C_{e}$ is the concentration of the adsorbate in the equilibrium solution $\left(\mathrm{moll}^{-1}\right) ; C_{\mathrm{ads}}$ is the amount of adsorbate retained on the adsorbent; $K_{L}$ is the maximum sorption at the experimental conditions, thus corresponding to the amount in the completed monolayer. The term $b$ includes various constants and nearly corresponds to an apparent adsorption equilibrium constant. As a consequence, $K_{L}$ greatly depends on the specific surface area while $b$ depends on the temperature.

The Langmuir isotherms for the adsorption of the Mo(VI)-calmagite complex are plotted in Fig. 1. The parameters $K_{L}$ and $b$ may be respectively assessed from the angular and linear coefficients of the $C_{e} / C_{\text {ads }}$ vs $C_{e}$ plot, at each temperature, and are collected in Table 2 . The decrease in $b$ with increasing temperatures suggests that the adsorption of the Mo(VI)-calmagite complex is an exothermic process. On the other hand, the values of $K_{L}$ indicate that, over the temperature range, only a low surface coverage

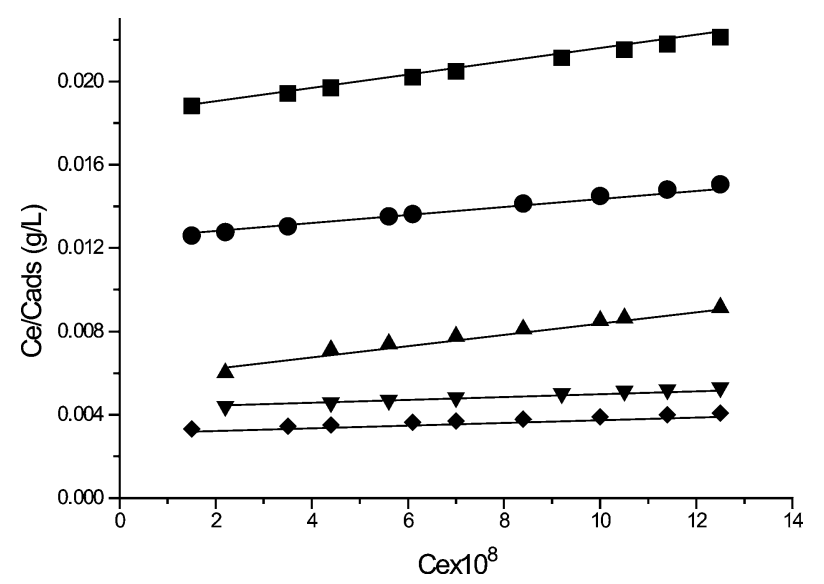

Fig. 1. Langmuir isotherms for the adsorption of Mo(VI)-calamagite complex onto active carbon, in the range 283-323 K: (ם) 323, ( $) 313,(\boldsymbol{\Delta})$ 303, ( $\mathbf{\nabla}) 298,(>) 283 \mathrm{~K}$. is attained, corresponding to nearly $0.6 \%$ of the maximum sorption, at $298 \mathrm{~K}$. This figure should be expected on the basis of the very dilute $\mathrm{Mo}(\mathrm{VI})$-calmagite complex solution and the high specific surface area of the active carbon.

The Freundlich isotherm is the most important multisite isotherm for rough surfaces [18] and is successfully used for the quantitative description of adsorption from solution,

$C_{\text {ads }}=K_{F} C_{e}^{1 / n}$,

where $K_{F}$ and $1 / n$ are fitting parameters that may be directly derived from a log-log plot of the adsorption data.

The Freundlich adsorption isotherm of the Mo(VI)calmagite complex onto active carbon, at $298 \mathrm{~K}$, is shown in Fig. 2. The related adsorption parameters, as determined from Eq. (5), were found to correspond to $K_{F}=(1.35 \pm$ $0.77) \times 10^{-3} \mathrm{molg}^{-1}$ and $1 / n=0.98 \pm 0.06$.

Equation (5) usually fits the data of solute adsorption from solution onto rough surfaces better than the Langmuir isotherm since it encompasses the surface heterogeneity and energy distribution of the adsorption sites. Nevertheless, the fitting parameters for the Freundlich equation are useful only for a narrow adsorbate concentration range. Moreover, as it does not predict any saturation coverage of the solid surface by the adsorbate, its predictive value is limited and the Freundlich model is actually discussed as an empirical expression to correlate experimental data [18].

In spite of the above discussion, there still remains a considerable effort in the literature [7-14,22-24] to get any

Table 2

Langmuir parameters from the $\mathrm{Mo}(\mathrm{VI})$-calmagite complex adsorption isotherms in the range $283-323 \mathrm{~K}$

\begin{tabular}{lcc}
\hline$T(\mathrm{~K})$ & $K_{L}\left(\mathrm{molg}^{-1}\right)$ & $b\left(\mathrm{lg}^{-1}\right)$ \\
\hline 283 & $(1.43 \pm 0.08) \times 10^{-4}$ & $(2.18 \pm 0.10) \times 10^{6}$ \\
298 & $(1.13 \pm 0.05) \times 10^{-4}$ & $(2.10 \pm 0.10) \times 10^{6}$ \\
303 & $(8.76 \pm 0.45) \times 10^{-5}$ & $(1.90 \pm 0.09) \times 10^{6}$ \\
313 & $(4.48 \pm 0.38) \times 10^{-5}$ & $(1.82 \pm 0.12) \times 10^{6}$ \\
323 & $(3.33 \pm 0.29) \times 10^{-5}$ & $(1.76 \pm 0.10) \times 10^{6}$ \\
\hline
\end{tabular}

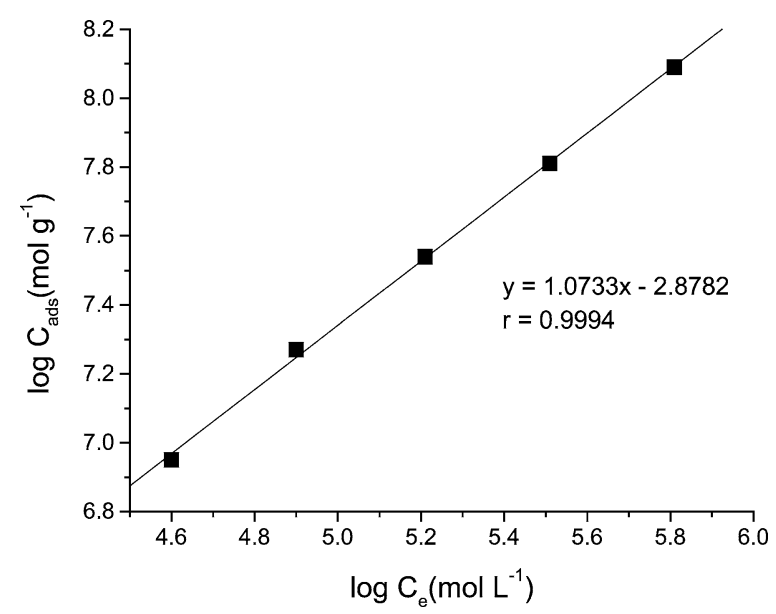

Fig. 2. Freundlich isotherm for the adsorption of Mo(VI)-calamagite complex onto active carbon, at $283 \mathrm{~K}$. 
physical interpretation to the Freundlich parameters. Saeed et al. [8-11] and Hasany et al. [12-15,22,23] interpreted $K_{F}$ as a relative indication of the adsorption capacity of an adsorbent toward a metal complex, for a narrow subregion having equally distributed energy sites. The $1 / n$ constant was then related to the nature and strength of the adsorption process or to the surface heterogeneity.

On the basis of the aforementioned assumptions [7-11, 22-24], the experimental data collected in the present paper were interpreted and the value found for $1 / n=0.98 \pm 0.06$, related to a rather homogeneous surface for the active carbon adsorbent [8,9]. According to Peña et al. [24], $1 / n<0.5$ is typical for hindered adsorption. Thus, for $1 / n=0.98$, one may speculate that the accessibility of the Mo(VI)calmagite complex to the pores of the active carbon was high and that the mass transfer limitation was not significant for the adsorption process. On the other hand, van der Waals interactions between the acid sites on the active carbon surface and the $\mathrm{Mo}(\mathrm{VI})$-calmagite complex should have favored the adsorption, accounting for a $K_{F}$ value as high as $(1.35 \pm 0.77) \times 10^{-3} \mathrm{molg}^{-1}$.

\subsection{Thermodynamic studies}

The thermodynamics of the Mo(VI)-calmagite complex adsorption onto active carbon was investigated in the range 298-315 K, applying the simplified treatment proposed by Saeed et al. [8,9] and Hasany et al. [12-14,22,23]. Thus, the values for standard enthalpy $\left(\Delta H^{0}\right)$, entropy $\left(\Delta S^{0}\right)$, Gibbs free energy $\left(\Delta G^{0}\right)$, and equilibrium constant $\left(K_{c}\right)$ were estimated using the equations

$\log K_{c}=-\frac{\Delta H^{0}}{2.303 R T}+\frac{\Delta S^{0}}{2.303 R}$,

$\Delta G^{0}=-R T \ln \left(K_{c}\right)$,

$K_{c}=\frac{C_{\text {ads }}}{C_{e}}$,

where $T$ is the absolute temperature $(\mathrm{K}), R$ is the ideal gas constant $\left(\mathrm{J} \mathrm{K}^{-1} \mathrm{~mol}^{-1}\right)$, and $C_{\text {ads }}$ and $C_{e}$ are, respectively, the equilibrium concentration of the $\mathrm{Mo}(\mathrm{VI})$-calmagite complex adsorbed onto the active carbon and in solution, at constant temperature.

The values for $K_{c}, \Delta H^{0} ; \Delta S^{0}$, and $\Delta G^{0}$ are collected in Table 3. The negative values found for the thermodynamic functions indicate that the adsorption process was spontaneous over the temperature range $\left(\Delta G^{0}\right)$, exothermic $\left(\Delta H^{0}\right)$, and accompanied by an entropy decrease $\left(\Delta S^{0}\right)$, as should be expected from the nature of the adsorption process. These figures also corroborate the previous assumptions made on the basis of the analysis of the Langmuir parameter $b$.

\subsection{Kinetic studies}

The kinetics of adsorption of the Mo(VI)-calmagite complex onto active carbon was investigated using the Lagergren
Table 3

Thermodynamic properties of the Mo(VI)-calmagite complex sorption onto active carbon

\begin{tabular}{lcccc}
\hline$T(\mathrm{~K})$ & $K_{c}$ & $\begin{array}{c}\Delta H^{0} \\
\left(\mathrm{~kJ} \mathrm{~mol}^{-1}\right)\end{array}$ & $\begin{array}{c}\Delta S^{0} \\
\left(\mathrm{~J} \mathrm{~mol}^{-1} \mathrm{~K}^{-1}\right)\end{array}$ & $\begin{array}{c}\Delta G^{0} \\
\left(\mathrm{KJ} \mathrm{mol}^{-1}\right)\end{array}$ \\
\hline 283 & 19.95 & -8.15 & -3.86 & -7.47 \\
298 & 17.18 & & & -7.18 \\
303 & 15.95 & & & -6.96 \\
313 & 14.12 & & & -6.79 \\
323 & 13.18 & & & -6.50 \\
\hline
\end{tabular}

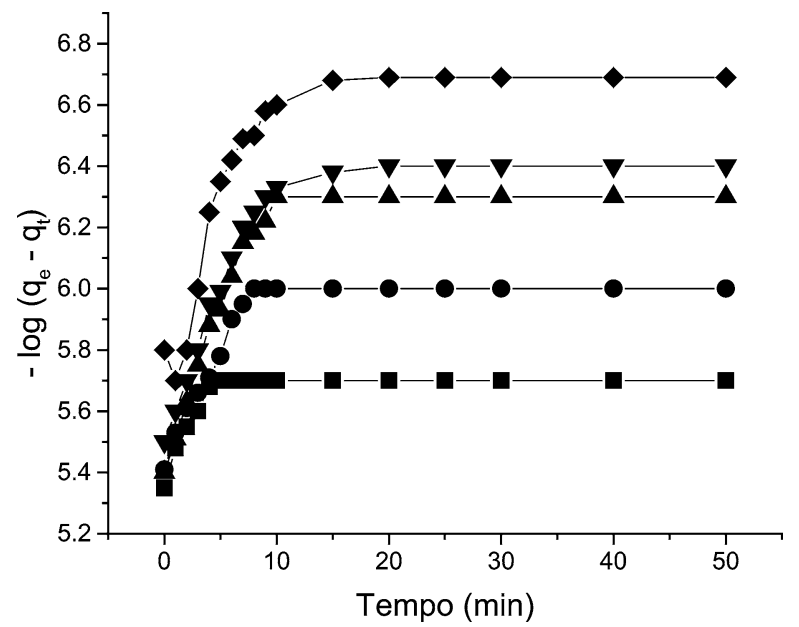

Fig. 3. Kinetics of $\mathrm{Mo}(\mathrm{VI})-$ calmagite complex adsorption on active carbon

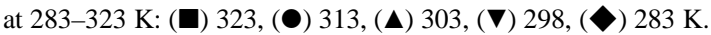

Table 4

Adsorption and desorption rate constants in the range 283-323 K

\begin{tabular}{lccc}
\hline$T(\mathrm{~K})$ & $K_{c}$ & $K_{t}$ & $K_{\text {dess }}$ \\
\hline 283 & 19.95 & 0.2032 & 0.1002 \\
298 & 17.18 & 0.2093 & 0.1022 \\
303 & 15.95 & 0.2120 & 0.1033 \\
313 & 14.12 & 0.2164 & 0.1053 \\
323 & 13.18 & 0.2229 & 0.1069 \\
\hline
\end{tabular}

criterion [12-14] for first order and adsorption time dependence given by the equation

$\log \left(q_{e}-q_{t}\right)=\log q_{e}-\frac{k_{t}}{2.303} t$,

where $q_{e}$ is the adsorption equilibrium concentration of the $\mathrm{Mo}(\mathrm{VI})$-calmagite complex onto active carbon, $q_{t}$ is its amount adsorbed at time $t$, and $k_{t}$ is the overall rate constant at a given temperature, $T$.

The plots of $\log \left(q_{e}-q_{t}\right)$ versus $t$ for the data obtained in the range $283-323 \mathrm{~K}$ at $\mathrm{pH} 2.0$ and for 40 min resulted in a series of curves, as shown in Fig. 3. Accordingly, this was interpreted as indicating that the adsorption process followed first-order kinetics up to the attainment of equilibrium at the applied experimental conditions. From the slope of each straight line corresponding to the first portion of the curves, the values for $k_{T}$ were determined and collected in Table 4. 


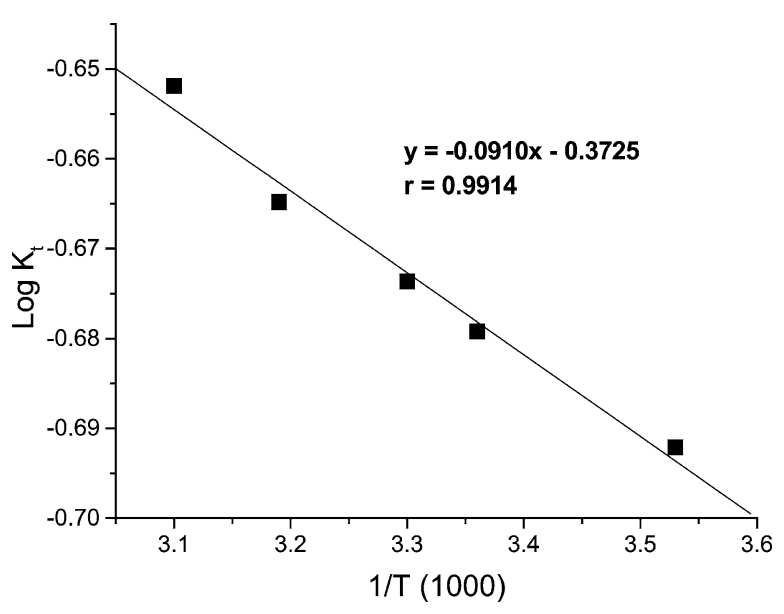

Fig. 4. Arrhenius plot for the adsorption of the Mo(VI)-calmagite complex onto active carbon at 283-323 K.

The activation energy for the adsorption process was then calculated using the Arrhenius equation,

$\log k_{T}=\log A-E_{a} / 2.303 R T$,

where $k_{T}$ is the rate constant a given temperature, $T, E_{a}$ is the activation energy for the overall adsorption process, and $A$ is the preexponential factor. Again, from the slope of the resulting straight line in the plot shown in Fig. 4, the value of the activation energy was calculated and found to be $E_{a}=1.7 \mathrm{~kJ} \mathrm{~mol}^{-1}$.

The desorption activation energy could be assessed using the relation

$E_{d}=\Delta H+E_{a}$.

The obtained value $E_{d}=9.8 \mathrm{~kJ} \mathrm{~mol}^{-1}$ was much higher than the activation energy value found for the adsorption path. Thus, these figures suggest that the overall process was kinetically limited by desorption of the Mo(VI)-calmagite complex, an energy-demanding path. In opposition, demanding less activation energy, the adsorption of the complex onto the active carbon surface was fast and fairly favored under the experimental conditions.

These assumptions may be further corroborated by comparing the adsorption and desorption rates, taking into consideration that $k_{a}$ nearly correspond to $k_{T}$ and that

$K_{c}=\frac{k_{a}}{k_{d}}$.

The obtained values, in the range of 283 to $323 \mathrm{~K}$, are also collected in Table 4.

\section{Conclusions}

On the basis of the thermodynamic and kinetic properties determined in the previous sections, one may conclude that active carbon is a very adequate solid material for preconcentration of trace molybdenum from solution, using calmagite as a complexing agent.

The high porous surface of the active coal led to very low surface coverage, thus allowing the proper application of the Langmuir adsorption model in the range of 283 to $323 \mathrm{~K}$.

\section{References}

[1] H.C. Santos, M.G.A. Korn, S.L.C. Ferreira, Anal. Chim. Acta 426 (2001) 79

[2] G.R. Boaventura, J.D.R. Hirson, R.E. Santelli, J. Braz. Chem. Soc. 6 (1995) 317.

[3] M.B.O. Giacomelli, J.B.B. da Silva, A.J. Curtius, Talanta 47 (1998) 877.

[4] T. Okutani, K. Noshiro, A. Sakuragawa, Anal. Sci. 14 (1998) 621.

[5] Q. Liu, X.Z. Liu, S.P. Zhou, Fenxi Shiyanshi 11 (1992) 16.

[6] J. Wei, Q. Liu, T. Okutani, Anal. Sci. 10 (1994) 465.

[7] T. Okutani, Y. Tsuruta, A. Sakuragawa, Anal. Chem. 65 (1993) 1273.

[8] M.M. Saeed, A. Rusheed, N. Ahmed, J. Tölgyessy, Sep. Sci. Technol. 29 (1994) 214.

[9] M.M. Saeed, S.M. Hasany, M. Ahmed, Talanta 50 (1999) 625.

[10] M.M. Saeed, A. Rusheed, N. Ahmed, J. Radioanal. Nucl. Chem. 211 (1996) 283.

[11] M.M. Saeed, A. Ghaffar, J. Radioanal. Nucl. Chem. 232 (1998) 171.

[12] S.M. Hasany, M.M. Saeed, M. Ahmed, Sep. Sci. Technol. 35 (2000) 379.

[13] S.M. Hasany, M.M. Saeed, M. Ahmed, J. Radioanal. Nucl. Chem. 246 (2000) 581.

[14] S.M. Hasany, M.M. Saeed, M. Ahmed, Talanta 54 (2001) 89.

[15] S.M. Hasany, M.M. Saeed, M. Ahmed, Sep. Sci. Technol. 36 (2001) 555.

[16] S.P. Mushran, O.M. Prakash, R. Murti, Analysis 3 (1975) 339.

[17] F.A.L. Dullien, Porous Media: Fluid Transport and Pore Structure, Academic Press, New York, 1979.

[18] R.I. Masel, Principles of Adsorption and Reaction on Solid Surfaces, Wiley, New York, 1996.

[19] C.H. Giles, D. Smith, J. Colloid Interface Sci. 47 (1974) 755.

[20] C.H. Giles, A.P. D'Silva, I.A. Easton, J. Colloid Interface Sci. 47 (1974) 766.

[21] J.H. De Boer, The Dynamical Character of Adsorption, Oxford Univ. Press, London, 1968.

[22] S.M. Hasany, M.M. Saeed, M. Ahmed, Sep. Sci. Technol. 34 (1999) 487.

[23] S.M. Hasany, M.M. Saeed, M. Ahmed, Radiochim. Acta 83 (4) (1998) 205-210.

[24] Y.P. De Peña, W. López, J.L. Burguera, M. Burguera, M. Gallignani, R. Brunetto, P. Carrero, C. Rondon, F. Imbert, Anal. Chim. Acta 403 (2000) 249. 